\title{
Interim FDG-PET Scan in Hodgkin's Lymphoma: Hopes and Caveats
}

\author{
M. André, ${ }^{1}$ T. Vander Borght, ${ }^{2}$ and A. Bosly ${ }^{3}$ \\ ${ }^{1}$ Department of Hematology, Grand Hôpital de Charleroi, Grand Rue, 3, 6000 Charleroi, Belgium \\ ${ }^{2}$ Department of Nuclear Medecine, Université Catholique de Louvain, Cliniques Universitaires UCL de Mont Godinne, Yvoir, Belgium \\ ${ }^{3}$ Department of Hematology, Université Catholique de Louvain, Cliniques Universitaires UCL de Mont Godinne, Yvoir, Belgium
}

Correspondence should be addressed to M. André, marc.andre@docs.be

Received 29 June 2010; Accepted 1 November 2010

Academic Editor: Emanuele Zucca

Copyright ( $) 2011$ M. André et al. This is an open access article distributed under the Creative Commons Attribution License, which permits unrestricted use, distribution, and reproduction in any medium, provided the original work is properly cited.

\begin{abstract}
FDG-PET has recently emerged as an important tool for the management of Hodgkins lymphoma. Although its use for initial staging and response evaluation at the end of treatment is well established, the place of interim PET for response assessment and subsequent treatment tailoring is still quite controversial. The use of interim PET after a few cycles of chemotherapy may allow treatment reduction for good responders, leading to lesser treatment toxicities as well as early treatment adaptation for bad responders with a potential higher chance for cure. Interpretation of interim PET is a rapidly moving field. Actually, visual interpretation is preferred over quantitative interpretation in this situation. The notion of minimal residual uptake emerged for faint persisting FDG uptake, but has evolved during the recent years. Guidelines using mediastinum and liver as references have been proposed at the expert meeting in Deauville 2009. Actually, several trials are ongoing both for localised and advanced disease to evaluate the FDG-PET potential for early treatment monitoring and tailoring. Until the results of these prospective randomized trials become available, treatment changes according to the interim PET results should remain inappropriate and limited to wellconducted clinical trials.
\end{abstract}

\section{Introduction}

This paper presents the latest evidence for the use and interpretation of early interim $18 \mathrm{~F}$-fluorodeoxyglucose positron emission tomography (PET) in classical Hodgkin's lymphoma (cHL).

The treatment of cHL is classically based on Ann Arbor staging.

Patients with limited disease (stage I-II) receive combined modality treatment mostly consisting of a few cycles of adriamycin, bleomycin, vinblastine and dacarbazine (ABVD), followed by involved-field radiotherapy 20-30 Gy [1]. This approach leads to very high event-free and overall survival (10-year overall survival estimates ranging from 84 to $97 \%$ ). However, these young patients experience late toxicities, such as secondary tumours and cardiac events, mostly related to radiotherapy, which lead to a delayed mortality [2]. Different randomised trials attempted to withdraw radiotherapy for unselected limited-disease patients. All of them showed reduced event-free survival for non irradiated patients [3-5]. Two recent GHSG (German Hodgkin Study Group) trials demonstrated that it is, nevertheless, possible to reduce the dose from 30 to $20 \mathrm{~Gy}$ in unselected patients with favourable disease [6, 7]. A concomitant expected decrease in late toxicities has not been observed yet, and our final goal remains to avoid radiotherapy for selected cHL patients.

Patients with advanced disease (bulky stage II with B symptoms and stages III-IV) receive generally $6-8$ cycles of chemotherapy, mainly ABVD or BEACOPPesc. Their 10year overall survival range from $75 \%$ to $85 \%$ [8]. If there are some evidence that BEACOPPesc is more effective than ABVD [9], this regimen also shows increased immediate (haematological) and delayed (fertility, myelodysplasia, acute myeloid leukemia) toxicities. It would be therefore, of major interest to be able to distinguish patients who can be cured with only a few cycles of BEACOPPesc or ABVD from patients who need a full course of 6-8 cycles of BEACOPPesc or even more aggressive treatments with highdose chemotherapy and stem cell transplantation upfront. 
Over the last decade, FDG-PET has become an important component in staging and end of treatment response assessment of patients with cHL [10]. When PET is combined with a CT scan (PET/CT), PET readings improve, actually PET/CT is the standard [11]. The adjunct of IV contrastenhanced CT is likely to enhance the benefit even further [12], but this is debated as other authors report that in patients undergoing PET/CT for staging or restaging after therapy of lymphoma, diagnostic CT with IV contrast does not add useful information regarding extent of lymphoma if the low-dose CT scan is interpreted individually [13]. The spread of PET/CT is rapidly expanding, and its use should be carefully evaluated. Its role to evaluate residual masses at the end of treatment is firmly established and has become a standard of care. For staging, FDG-PET leads 15\%-20\% and $5 \%-15 \%$ changes in stage and treatment, respectively [14]. The impact of these treatment modifications has, however, never been evaluated prospectively. The use of FDG-PET for radiotherapy planning in Stage I or II cHL may induce significant irradiated field modifications, but today there are no validated guidelines to integrate such information in the treatment planning $[15,16]$. Finally, FDG-PET might be used during therapy as a predictor to treatment response.

\section{Interim PET As Predictor of Relapse}

In two preliminary prospective studies, Hutchings et al. $[17,18]$ showed that patients with advanced $\mathrm{cHL}$ who were PET positive after 2 ABVD had a 2 -year PFS of $0 \%-6 \%$ by contrast to $94 \%$ for those with PET negativeresult. Those two cohorts were joined and expanded $(n=260)$ to report the same results [19]. The prognostic value of the interim PET completely overshadowed the International Prognostic Score (IPS).

In a study of 41 patients including $23 \mathrm{cHL}$, Kostakoglu et al. performed FDG-PET soon after 1 cycle of chemotherapy and showed a 2-year progression-free survival for PETnegative patients after 1 cycle of therapy of $100.0 \%$, compared with only $12.5 \%$ (95\% CI: $2.1-32.8$ ) in those with a positive result [20]. The timing of treatment assessment may be critical, especially to distinguish patients refractory to first-line therapy from those who relapse at a later time. The former may benefit from very early alternative therapy avoiding the complications of continued ineffective therapy. Likewise, the identification of patients who are likely to be cured by the first-line therapy may offer the potential of shortening the duration or intensity of treatment.

In a recently published metaanalysis, Terasawa [21] showed that in 360 advanced cHL, FDG-PET for interim response assessment had an overall sensitivity of $0.81(95 \%$ CI: 0.72-0.89) and a specificity of 0.97 (95\% CI: 0.94-0.99). Because of a 28.4 positive likelihood ratio, positive FDG-PET results after a few cycles of chemotherapy would probably have an excellent ability to predict poor responders in advanced adult cHL. Meta-regression and subgroup analysis did not identify factors that affect prognosis accuracy.

The positive predictive value (PPV) of FDG-PET in localised $\mathrm{cHL}$ is less firmly established. In the study of
Hutchings et al. [22], a significant proportion of interim PET-positive patients did not relapse. In order to identify the group of patients at very high risk for relapse more accurately, they analysed separately the patients with stage III and stage III-IV. The differences in PFS between stage I-II positive and negative was significant, but only $2 / 7$ interim PET-positive patients with early stage have relapsed in contrast to advanced-stage patients who all relapsed within 2 years in the same circumstances. More recently, Sher et al. reported that only $3 / 20$ patients with positive interim FDGPET relapse in a study population of, mostly stage I-II, cHL patients. This especially 15\% PPV was, however, obtained in the context of a consolidative radiotherapy given to all patients (PET positive and negative) [23].

\section{Interpretation of Interim PET}

3.1. General Considerations. In $\mathrm{cHL}$, the Hodgkin or ReedSternberg cells represents less than $1 \%$ of the lymph node population. They are surrounded by a large number of mononuclear cells that are very metabolic and responsible for FDG uptake in vivo. The metabolic viability of such environment works as an amplifier for the FDG-PET signal, but might also become negative despite the persistence of a large tumoral mass.

This anatomopathological aspect is quite different from that observed in non-Hodgkin lymphoma where neoplastic cells account for $90 \%$ of the lymph node population. Therefore, the timing for interim FDG-PET interpretation and the guidelines might differ largely between $\mathrm{cHL}$ and NHL, which own different biologic behaviour and response profiles.

If the predictive value of FDG-PET depends on the type of lymphoma, the type of chemotherapy used may also be of major significance. Most of the patients included in the studies were treated using ABVD. Until recently, the prognostic value of interim FDG-PET in the context of BEACOPP regimens in advanced cHL patients was not established. Avigdor et al. [24] reported a relatively high negative predictive value (NPV; 87\%), but a much lower PPV (45\%), for interim FDG-PET carried out after two cycles of BEACOPPesc before decreasing therapy to ABVD. These findings were indeed different from those observed during ABVD treatment. Nevertheless, the results of early FDG-PET still maintained a significant long-term prognostic role in terms of PFS presumably due to the relatively high NPV. Similarly, Gallamini et al. [25] showed that PET carried out after two cycles of BEACOPPesc retained its long-term prognostic role despite a PPV of $60 \%$, which was also much lower than expected.

3.2. Visual Interpretation and Minimal Residual Uptake $(M R U)$. In $10 \%$ of the patients undergoing early FDGPET, a persisting faint residual FDG uptake is recorded, most often in a site with previous bulky disease. Hutchings et al. described this new grey-zone as minimal residual disease (MRU) and defined it as a FDG uptake just above background, which is unlikely to represent persisting $\mathrm{cHL}$ 
TABLE 1: Score 3 might be either considered as FDG-PET positive when a therapy decrease is planned in localised $\mathrm{cHL}$ or negative when treatment intensification is planned in advanced cHL.

\begin{tabular}{l}
\hline Five Point scale. \\
\hline (1) No uptake \\
(2) Uptake $\leq$ mediastinum \\
(3) Uptake $>$ mediastinum but $\leq$ liver \\
(4) Uptake moderately more than liver uptake, at any site \\
(5) Markedly increased uptake at any site and new site of disease.
\end{tabular}

[22]. MRU probably represents an inflammatory reaction consecutive to chemotherapy with a nonspecific uptake of FDG. However, the boundaries of the MRU concept have evolved during the recent years. Gallamini et al. [19] considered MRU as a weakly persisting uptake with an intensity equal or slightly more than the mediastinal blood pool. In 2008, Barrington et al. proposed a residual uptake with an intensity lower or equal to the intensity of the liver [26]. The goal of the evolution of MRU definition is to reduce the false positive report. The consequence of such evolution is, however, that different MRU definitions are used among several trials making comparison extremely hazardous.

3.3. Quantitative Interpretation. Besides visual inspection of PET images, semiquantitative analyses using standardized uptake values (SUVs) allow for an objective assessment of treatment response, thereby eliminating observer variation and providing the opportunity to reduce the grey-zone by adding the quantitative power of PET.

However, for multicentre studies, the comparison of SUV results obtained in different centres is still hampered by the wide variability in the methodology of data acquisition, image reconstruction, and data analysis procedures. Therefore, protocols are established for standardisation and quantification of PET [27]. The quantitative assessment of tumour responses and the comparison among studies require rigorous quality control, especially if performed on different PET systems or using different protocols, and because of variation among institutions [28].

Current guidelines suggest that a visual assessment of PET status is adequate and sufficient for a positive or negative decision after completion of therapy; however, during treatment or in clinical trials, some form of semiquantitation may be helpful [29-31]. Cut-off levels of SUV to determine response to therapy are also likely to be dependent on tumour and treatment type, and so, need to be evaluated in further prospective clinical trials.

3.4. Proposed Guidelines for Interpretation. With the demonstration of interim FDG-PET prognostic value in cHL, different trials have been initiated to use such information for individual treatment adaptation. A standardization effort of FDG-PET interpretation criteria seems particularly relevant for a potential extension of the method on a worldwide basis.

In 2007, the International Harmonization Project (IHP) subcommittee developed consensus recommendations in the
TABLE 2: Example of cut-off for therapy (a) decrease in localised cHL, (b) increase in advanced cHL.

(a)
(1) No uptake
(2) Uptake $\leq$ mediastinum
(3) Uptake $>$ mediastinum but $\leq$ liver
(4) Uptake moderately more than liver uptake, at any site
(5) Markedly increased uptake at any site and new site of disease.

(b)
(1) No uptake
(2) Uptake $\leq$ mediastinum
(3) Uptake $>$ mediastinum but $\leq$ liver
(4) Uptake moderately more than liver uptake, at any site
(5) Markedly increased uptake at any site and new site of disease.

use of FDG-PET in lymphoma based on the literature and the collective expertise of its members [29]. Visual assessment alone was considered adequate for FDG-PET reading after the completion of therapy. Mediastinal blood pool activity was recommended as the reference background activity to define FDG-PET positivity for a residual mass $\geq 2 \mathrm{~cm}$ in greatest transverse diameter, regardless of its location. A smaller residual mass or a normal-sized lymph node should be considered positive if its activity is above that of the surrounding background. Specific criteria for defining FDGPET positivity in the liver, spleen, lung, and bone marrow were also proposed. Use of attenuation-corrected PET was strongly encouraged.

Two drawbacks can be considered for the use of IHP criteria in interim FDG-PET for cHL. First, these criteria were developed for interpretation of FDG-PET at the end of treatment and not specifically for interim FDG-PET. Moreover, the aim of PET scan performed at the end of treatment is different from that of interim scan: the first is aimed at assessing the response, the second at assessing chemosensitivity. For the former the gold standard reference is the biopsy to demonstrate CR or non-CR, for the second the gold standard does not exist, at the moment.

Second, these criteria use the lymph node size as cut-off for reference background (i.e.: when $\geq 2 \mathrm{~cm}$ : background is the mediastinal blood pool activity, whereas $<2 \mathrm{~cm}$ it is the surrounding background). As a recent radiological study has shown the reproducibility of lymph nodes, measurements is the lowest between 15 to $20 \mathrm{~mm}$, a range which might lead to major discrepancies in FDG-PET interpretation [32] as the reference background changes. In 2009, an international workshop on interim FDG-PET [33] took place in Deauville, France, to reach a consensus on simple and reproducible criteria for interim FDG-PET. The experts proposed for $\mathrm{cHL}$ that (1) a baseline FDG-PET/CT should be performed prior to therapy initiation, (2) and that a visual analysis using a five point-scale should be applied (Table 1), and (3) for 
TABLE 3

\begin{tabular}{lccc}
\hline Name of study & Study Group & Classification & PET intervention \\
\hline 20051 (H10) & EORTC-GELA-IIL & Localized & No radiotherapy PET- after 2 ABVD \\
RAPID & UK NCRI lymphoma group & Localized & No radiotherapy PET- after 3 ABVD \\
HD16 & GHSG & Localized & No radiotherapy PET- after 2 ABVD \\
PET adapted chemo & GITIL & Advanced & BEACOPPesc if PET+ after 2 ABVD \\
RATHL & UK NCRI lymphoma group & Advanced & BEACOPPesc if PET+ after 2 ABVD \\
HD0801 & IIL & Advanced & Salvage if PET+ after 2 ABVD \\
HD18 & GHSG & Advanced & BEACOPPesc reduced to 4 cycles if PET2- \\
AHL2011 & GELA & Advanced & BEACOPPesc reduced to ABVD if PET2- \\
Avigdor et al. [24] & Israel & Advanced & Reduce to 4 ABVD if PET- after 2 BEACOPPesc \\
\hline
\end{tabular}

the therapeutic decision, the cut-off should be determined according to the strategy (Tables 2(a) and 2(b)). A cohort of ABVD-treated HL patients was collected with the aim to validate the proposed criteria. This International validation study is currently under progress.

Finally, the use of dynamic visual score, which combines the metabolic evolution of each disease site has been recently reported to be superior to the binary static scores [34].

3.5. FDG-PET Review System. Despite all these efforts of reading standardisation, many difficulties arise when minimal residual uptake is present. Even with blind assessment, readers may disagree especially with borderline or complex cases. Interpretation of FDG-PET is subject to a number of variables, including the reader experience. Zijlstra et al. looked at the scoring of 11 nuclear medicine physicians. They compared their results with those of an expert interpreter. The agreement was $82 \%-94 \%$ when the expert reading was PET-positive, but only $45 \%$ when it was PET negative. The same group also showed that more experienced readers tended to have fewer false positives, demonstrating the need for standardization [35].

Especially in the context of clinical trials, it is highly desirable to establish a reading procedure that synthesizes the opinions of several experts, potentially from different imaging departments, for reducing the impact of inter observer variability. A major limitation to this approach is the necessity to obtain the multiple interpretations in a clinically relevant time frame (typically less than 72 hours) in order to modify the therapeutic strategy, if needed, during the chemotherapy regimen. For these reasons, the classic retrospective or local blinded independent central review is not applicable.

The development of such network has been recently developed by the GELA group [36]. The cornerstone of the network is a multimodality workstation which allows side-by-side display of pre- and post-treatment FDG$\mathrm{PET} /$ computed tomography (CT), as well as complete image processing, including standardized uptake values analysis. A central server dispatches the raw data to the workstations of experts. They make their own independent image processing and interpretation, and send the optical scan report form with the result to the central server where an integrated computation of the interpretations is performed. From June 2007 to November 2008, FDG-PET from 166 consecutive patients included in the H10 study were reviewed. Six percent discordant PET2 readings were observed between the local site reader and the central review panel, $73 \%$ of them were modified from negative to positive. More importantly, a significantly higher inter-observer agreement $(P \leq .0001)$ was achieved when the readers could interpret side by side the PET2 with baseline FDG-PET.

Another network was also established in the United Kingdom for clinical trial in cHL. This network has been set up with a "core laboratory" to coordinate quality control and interpret scans. Images are exchanged via web on a dedicated website. Recently, a good agreement was reported within this network in a series of 44 patients with stage II-IV cHL treated with ABVD (Kappa: 0.85 (95\% CI 0.74-0.96)) [37].

\section{Ongoing Studies}

Different trials evaluating FDG-PET response-adapted therapy were recently initiated (Table 3 ). In localised $\mathrm{cHL}$, all evaluate omission of radiotherapy for FDG-PET-negative patients after 2-3 cycles of ABVD. In the EORTC-GELAIIL trial, early modification of chemotherapy for PETpositive patients has also been a secondary objective. In advanced cHL, two different strategies have been applied: either patients who are PET-positive after 2 ABVD will receive more aggressive chemotherapy or intensification (escaladating strategy) or, in three other trials, patients who are PET-negative after $2 \mathrm{BEACOPPesc}$ receive less cycles of regimen or switch to ABVD (reducing strategy).

\section{Conclusions}

Prognostic accuracy studies of interim FDG-PET for advanced-stage HL have consistently reported excellent specificity and moderately good sensitivity.

The reported prognostic accuracy is reasonably applicable to low- or intermediate-risk advanced-stage HL patients undergoing ABVD first-line therapy. It is unclear whether the accuracy can also be applied to high-risk patients in whom BEACOPPesc is used. Moreover, the interpretation of interim FDG-PET is still the subject of debate and controversies, so that no consensus is currently reached. 
Thus, the additional prognostic value of interim FDGPET in the current management strategies is still unclear and its use should still be reserved for research settings.

\section{References}

[1] C. Fermé, H. Eghbali, J. H. Meerwaldt et al., "Chemotherapy plus involved-field radiation in early-stage Hodgkin's disease," New England Journal of Medicine, vol. 357, no. 19, pp. 19161927, 2007.

[2] O. Favier, N. Heutte, A. Stamatoullas-Bastard et al., "Survival after Hodgkin lymphoma: causes of death and excess mortality in patients treated in 8 consecutive trials," Cancer, vol. 115, pp. 1680-1691, 2009.

[3] D. J. Straus, C. S. Portlock, J. Qin et al., "Results of a prospective randomized clinical trial of doxorubicin, bleomycin, vinblastine, and dacarbazine (ABVD) followed by radiation therapy (RT) versus ABVD alone for stages I, II, and IIIA nonbulky Hodgkin disease," Blood, vol. 104, no. 12, pp. 34833489, 2004.

[4] R. M. Meyer, M. K. Gospodarowicz, J. M. Connors et al., "Randomized comparison of ABVD chemotherapywith a strategy that includes radiation therapy inpatients with limited-stage Hodgkin's lymphoma: National Cancer Institute of Canada Clinical Trials Group and the Eastern Cooperative Oncology group," Journal of Clinical Oncology, vol. 23, pp. 4623-4642, 2005.

[5] H. Eghbali, P. Brice, G. Y. Cremers et al., "Comparison of three radiation dose levels after EBVP regimen in favorable supradiaphragmatic clinical stages (CS) I-II Hodgkin's Lymphoma (HL): preliminary results of the EORTC-GELA H9-F trial," Blood, vol. 106, article 814, 2005.

[6] A. Engert, A. Pluetschow, H. T. Eicht et al., "Reduced intensity treatment in Hodgkin's lymphoma," The New England Journal of Medicine, vol. 343, pp. 640-652, 2010.

[7] H. T. Eich, V. Diehl, H. Goergen et al., "Intensified chemotherapy and dose-reduced involved-field radiotherapy in patients with early unfavourable Hodgkin lymphoma: final analysis of the German Hodgkin Study Group HD11 trial," Journal of Clinical Oncology, vol. 28, pp. 4199-4206, 2010.

[8] A. Engert, V. Diehl, J. Franklin et al., "Escalated-dose BEACOPP in the treatment of patients with advanced-stage Hodgkin's lymphoma: 10 years of follow-up of the GHSG HD9 study," Journal of Clinical Oncology, vol. 27, no. 27, pp. 4548-4554, 2009.

[9] M. Federico, S. Luminari, E. Iannitto et al., "ABVD compared with BEACOPP compared with CEC for the initial treatment of patients with advanced hodgkin's lymphoma: results from the HD2000 gruppo italiano Per lo studio dei linfomi trial," Journal of Clinical Oncology, vol. 27, no. 5, pp. 805-811, 2009.

[10] L. Specht, "2-[18F]fluoro-2-deoxyglucose positron-emission tomography in staging, response evaluation, and treatment planning of lymphomas," Seminars in Radiation Oncology, vol. 17, no. 3, pp. 190-197, 2007.

[11] M. Allen-Auerbach, A. Quon, W. A. Weber et al., "Comparison between 2-deoxy-2-[F]fluoro-D-glucose positron emission tomography and positron emission tomography/computed tomography hardware fusion for staging of patients with lymphoma," Molecular Imaging and Biology, vol. 6, no. 6, pp. 411-416, 2004.

[12] N. G. Schaefer, T. F. Hany, C. Taverna et al., "Non-Hodgkin lymphoma and Hodgkin disease: coregistered FDG PET and $\mathrm{CT}$ at staging and restaging-do we need contrast-enhanced CT?" Radiology, vol. 232, no. 3, pp. 823-829, 2004.
[13] R. L. Elstrom, J. P. Leonard, M. Coleman, and R. K. J. Brown, "Combined PET and low-dose, noncontrast CT scanning obviates the need for additional diagnostic contrast-enhanced CT scans in patients undergoing staging or restaging for lymphoma," Annals of Oncology, vol. 19, no. 10, pp. 17701773, 2008.

[14] P. Seam, M. E. Juweid, and B. D. Cheson, "The role of FDGPET scans in patients with lymphoma," Blood, vol. 110, no. 10, pp. 3507-3516, 2007.

[15] M. Hutchings, A. Loft, M. Hansen, A. K. Berthelsen, and L. Specht, "Clinical impact of FDG-PET/CT in the planning of radiotherapy for early-stage Hodgkin lymphoma," European Journal of Haematology, vol. 78, no. 3, pp. 206-212, 2007.

[16] P. Pommier, S. Dussart, T. Girinsky et al., "Impact of 18F-fluoro-2-deoxyglucose positron emission tomography on treatment strategy and radiotherapy planning for stage I-II Hodgkin disease: a prospective multicenter study," International Journal of Radiation Oncology, Biology, Physics. In press.

[17] M. Hutchings, A. Loft, M. Hansen et al., "FDG-PET after two cycles of chemotherapy predicts treatment failure and progression-free survival in Hodgkin lymphoma," Blood, vol. 107, no. 1, pp. 52-59, 2006.

[18] A. Gallamini, L. Rigacci, F. Merli et al., "The predictive value of positron emission tomography scanning performed after two courses of standard therapy on treatment outcome in advanced stage Hodgkin's disease," Haematologica, vol. 91, no. 4, pp. 475-481, 2006.

[19] A. Gallamini, M. Hutchings, L. Rigacci et al., "Early interim 2[F] fluoro-2-deoxy-D-glucose positron emission tomography is prognostically superior to international prognostic score in advanced-stage Hodgkin's lymphoma: a report from a joint Italian-Danish study," Journal of Clinical Oncology, vol. 25, no. 24, pp. 3746-3752, 2007.

[20] L. Kostakoglu, S. J. Goldsmith, J. P. Leonard et al., "FDG-PET after 1 cycle of therapy predicts outcome in diffuse large cell lymphoma and classic Hodgkin disease," Cancer, vol. 107, no. 11, pp. 2678-2687, 2006.

[21] T. Terasawa, J. Lau, S. Bardet et al., "Fluorine-18fluorodeoxyglucose positron emission tomography for interim response assessment of advanced-stage Hodgkin's lymphoma and diffuse large B-cell lymphoma: a systematic review," Journal of Clinical Oncology, vol. 27, no. 11, pp. 1906-1914, 2009.

[22] M. Hutchings, N. G. Mikhaeel, P. A. Fields, T. Nunan, and A. R. Timothy, "Prognostic value of interim FDG-PET after two or three cycles of chemotherapy in Hodgkin lymphoma," Annals of Oncology, vol. 16, no. 7, pp. 1160-1168, 2005.

[23] D. J. Sher, P. M. Mauch, A. Van Den Abbeele, A. S. LaCasce, J. Czerminski, and A. K. Ng, "Prognostic significance of mid- and post-ABVD PET imaging in Hodgkin's lymphoma: the importance of involved-field radiotherapy," Annals of Oncology, vol. 20, no. 11, pp. 1848-1853, 2009.

[24] A. Avigdor, S. Bulvik, I. Levi et al., "Two cycles of escalated BEACOPP followed by four cycles of ABVD utilizing earlyinterim PET/CT scan is an effective regimen for advanced high-risk Hodgkin's lymphoma," Annals of Oncology, vol. 21, no. 1, pp. 126-132, 2010.

[25] A. Gallamini, S. Viviaru, V Bonfante et al., "Early interim FDG-PET during intensified BEACOPP therapy for advanced stage Hodgkin disease shows a lower predictive value than during ABVD," Haematologica, vol. 92, pp. 143-144, 2007.

[26] S. F. Barrington, W. Qian, E. J. Somer et al., "Concordance between four European centres of PET reporting criteria designed for use in multicentre trials in Hodgkin lymphoma," 
European Journal of Nuclear Medicine and Molecular Imaging, vol. 36, supplement 2, p. S252, 2009.

[27] R. Boellaard, W. J. G. Oyen, C. J. Hoekstra et al., "The Netherlands protocol for standardisation and quantification of FDG whole body PET studies in multi-centre trials," European Journal of Nuclear Medicine and Molecular Imaging, vol. 35, no. 12, pp. 2320-2333, 2008.

[28] R. Boellaard, M. J. O’Doherty, W. A. Weber et al., "FDG PET and PET/CT: EANM procedure guidelines for tumour PET imaging: version 1.0," European Journal of Nuclear Medicine and Molecular Imaging, vol. 37, no. 1, pp. 181-200, 2010.

[29] M. E. Juweid, S. Stroobants, O. S. Hoekstra et al., "Use of positron emission tomography for response assessment of lymphoma: consensus of the imaging subcommittee of international harmonization project in lymphoma," Journal of Clinical Oncology, vol. 25, no. 5, pp. 571-578, 2007.

[30] L. K. Shankar, J. M. Hoffman, S. Bacharach et al., "Consensus recommendations for the use of F-FDG PET as an indicator of therapeutic response in patients in National Cancer Institute Trials," Journal of Nuclear Medicine, vol. 47, no. 6, pp. 10591066, 2006.

[31] C. Lin, E. Itti, C. Haioun et al., "Early F-FDG PET for prediction of prognosis in patients with diffuse large Bcell lymphoma: SUV-based assessment versus visual analysis," Journal of Nuclear Medicine, vol. 48, no. 10, pp. 1626-1632, 2007.

[32] M. Fabel, H. von Tengg-Kobligk, F. L. Giesel et al., "Semiautomated volumetric analysis of lymph node metastases in patients with malignant melanoma stage III/IV-A feasibility study," European Radiology, vol. 18, no. 6, pp. 1114-1122, 2008.

[33] M. Meignan, A. Gallamini, M. Meignan, A. Gallamini, and C. Haioun, "Report on the first international workshop on interim-PET-scan in lymphoma," Leukemia \& Lymphoma, vol. 50, no. 8, pp. 1257-1260, 2009.

[34] E. J. Dann, R. Bar-Shalom, A. Tamir et al., "A functional dynamic scoring model to elucidate the significanceof postinduction interim fluorine-18-fluorodeoxyglucose positron emission tomography findings in patients with hodgkin's lymphoma," Haematologica, vol. 95, no. 7, pp. 1198-1206, 2010.

[35] J. M. Zijlstra, E. F. Comans, A. Van Lingen et al., "FDG PET in lymphoma: the need for standardization of interpretation. An observer variation study," Nuclear Medicine Communications, vol. 28, no. 10, pp. 798-803, 2007.

[36] M. Meignan, E. Itti, S. Bardet et al., "Development and application of a real-time on-line blinded independent central review of interim pet scans to determine treatment allocation in lymphoma trials," Journal of Clinical Oncology, vol. 27, no. 16, pp. 2739-2741, 2009.

[37] S. F. Barrington, W. Qian, E. J. Somer et al., "Concordance between four European centres of PET reporting criteria designed for use in multicentre trials in Hodgkin lymphoma," European Journal of Nuclear Medicine and Molecular Imaging, vol. 37, pp. 1824-1833, 2010. 


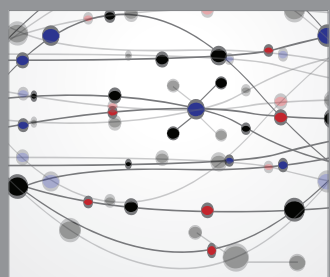

The Scientific World Journal
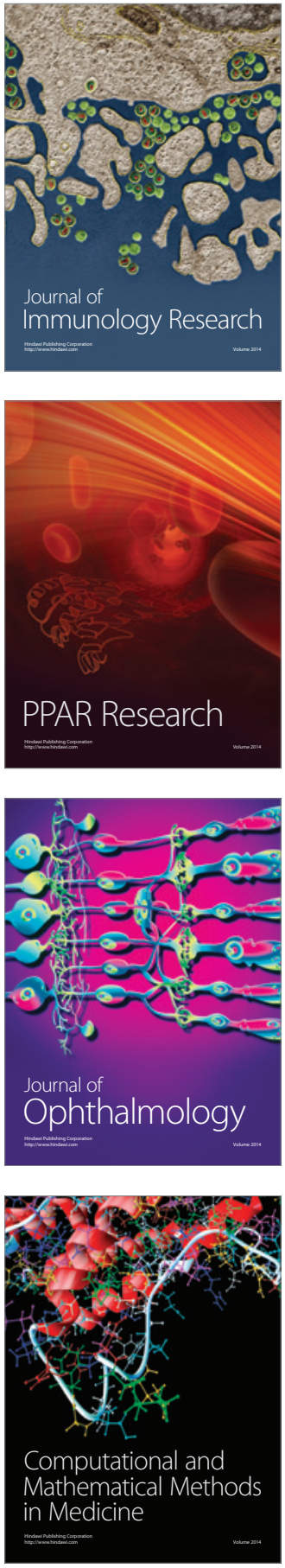

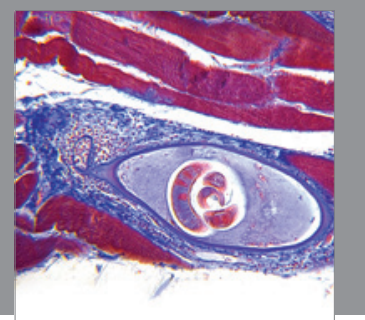

Gastroenterology

Research and Practice
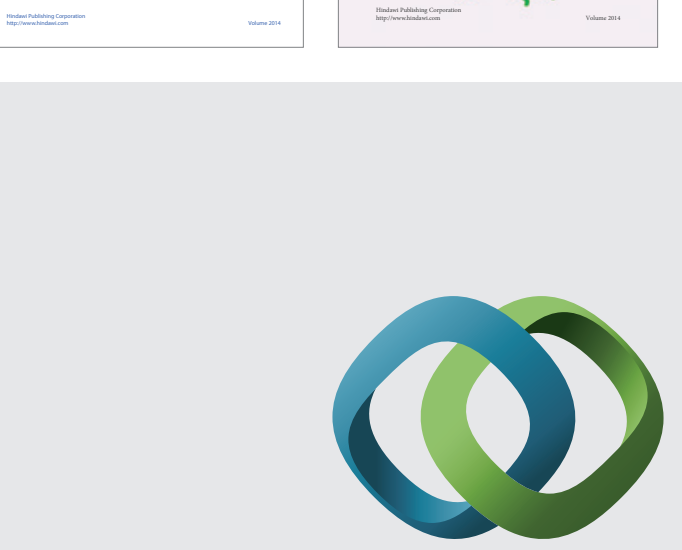

\section{Hindawi}

Submit your manuscripts at

http://www.hindawi.com
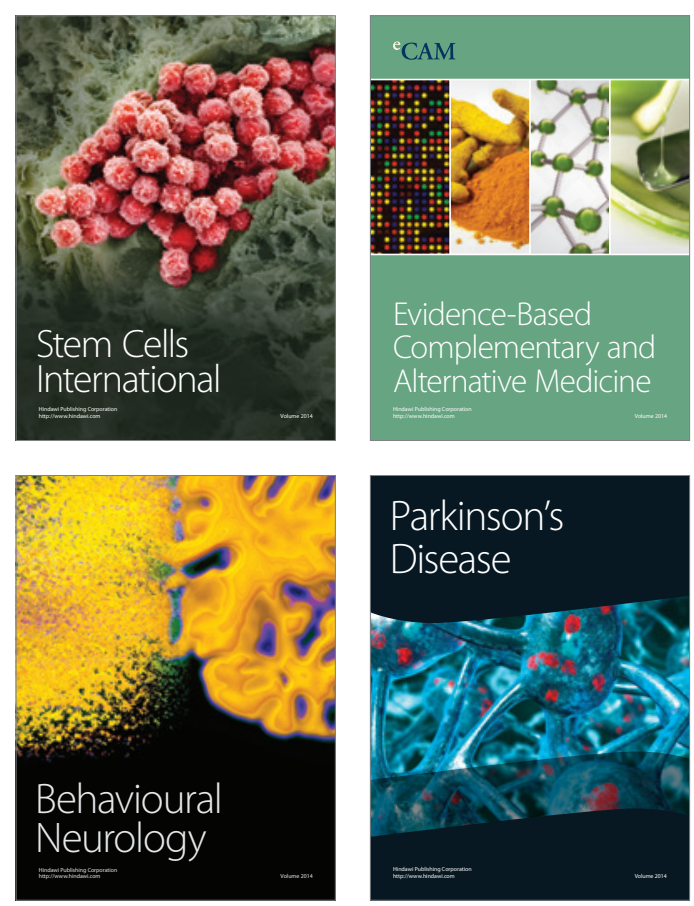

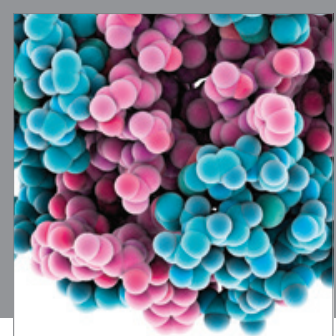

Journal of
Diabetes Research

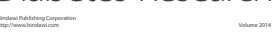

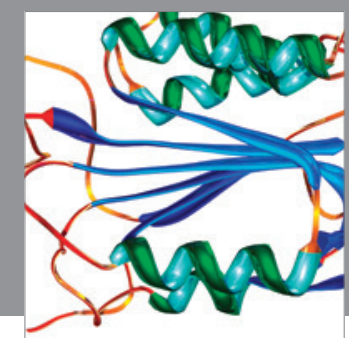

Disease Markers
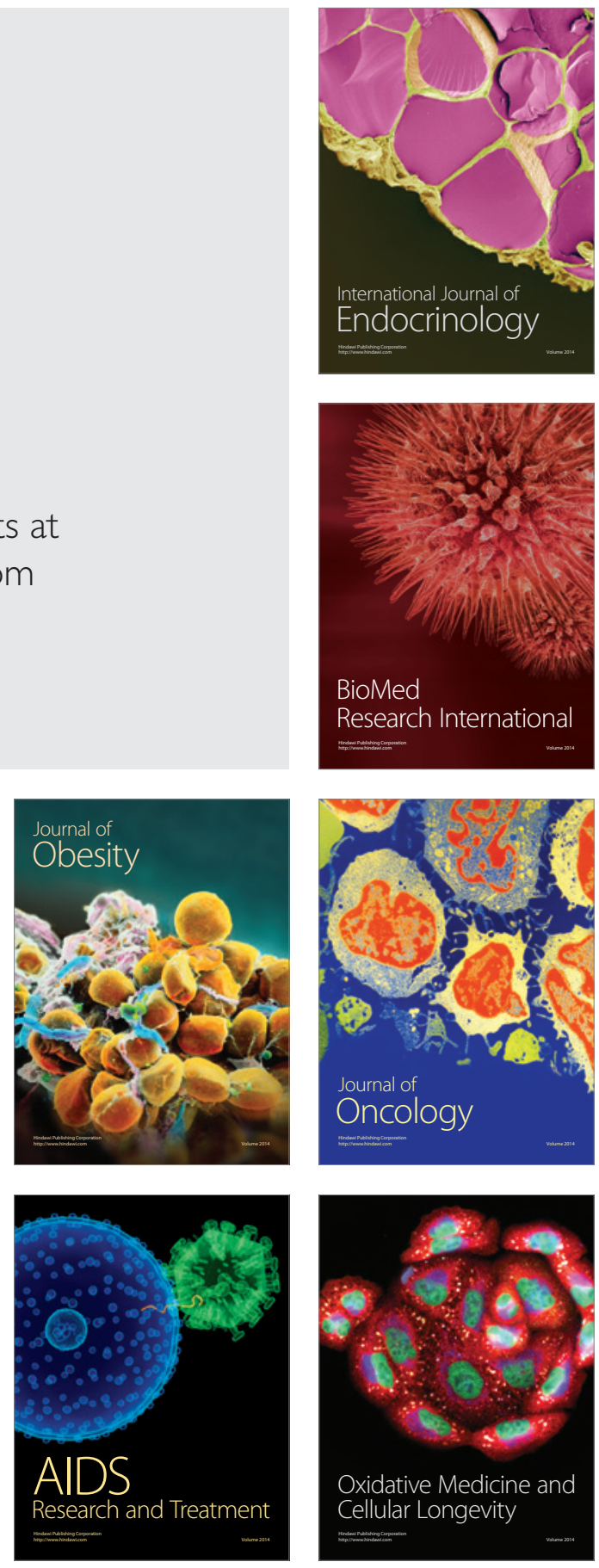